\title{
Pain in the management of opioid use disorder
}

\author{
Sunil Sirohi' \\ Amit K Tiwari ${ }^{2}$ \\ 'Laboratory of Endocrine and \\ Neuropsychiatric Disorders, Division \\ of Basic Pharmaceutical Sciences, \\ College of Pharmacy, Xavier \\ University of Louisiana, New Orleans, \\ LA, ${ }^{2}$ Department of Pharmacology and \\ Experimental Therapeutics, College \\ of Pharmacy and Pharmaceutical \\ Sciences, University of Toledo, Toledo, \\ $\mathrm{OH}$, USA
}

This article was published in the following Dove Press journal:

Journal of Pain Research

4 November 2016

Number of times this article has been viewed

Opioids remain the drug of choice for the clinical management of moderate to severe pain. However, in addition to their most effective analgesic actions, opioids also produce a sense of well-being and euphoria, which may trigger significant concerns associated with their use. ${ }^{1}$ In fact, there has been an alarming increase in prescription opioid use, abuse and illicit use; and according to the National Center for Health Statistics, the total number of deaths related to opioid overdose has more than tripled from 2011 to $2014 . .^{2-5}$ Although representing $5.0 \%$ of the global population, studies report that Americans consume $80 \%$ of the global opioid supply, ${ }^{3}$ and the United States is experiencing an opioid abuse epidemic. ${ }^{6}$ Considering this unprecedented rise in opioid consumption, the United States Centers for Disease Control and Prevention has listed prescription opioid overdose among one of the 10 most important public health problems in all the 50 states. $^{7}$

Opioid use disorder (OUD) is defined as a repeated manifestation of 2 or more of 11 problems (e.g., strong desire to use an opioid, use of an opioid in increased amounts, continued opioid use despite negative consequences and withdrawal when the dose of an opioid is decreased) within a 12-month period. ${ }^{8}$ Recent studies report a shocking increase in emergency room visits and individuals seeking treatment for OUD. ${ }^{9}$ Various pharmacotherapies exist for the detoxification or long-term management of OUD, including opioid antagonists (naltrexone), partial agonists (buprenorphine), opioid agonists (methadone), alpha-2 adrenergic agonists (clonidine) and psychosocial interventions. A detailed account of these interventions is reviewed elsewhere. ${ }^{10,11}$ While these medications have better efficacy in the management of OUD compared to non-medication interventions, treatment requires long-term management, and a high rate of relapse is a significant concern posttreatment. . $^{10,12-14}$

A growing body of literature suggests a higher prevalence of chronic pain in OUD patients, and approximately $50 \%$ of patients maintained on methadone or buprenorphine have been reported to experience chronic pain. ${ }^{15-18}$ In addition, heightened psychiatric problems are associated with increased pain intensity in OUD patients receiving opioid agonist-assisted treatment (OAAT) ${ }^{16}$ Furthermore, in patients receiving OAAT, chronic pain is associated with poor abstinence rate, ${ }^{19,20}$ however, no association has been reported by others. ${ }^{21,22}$ Drug craving, defined as an intense urge for a drug experienced previously, ${ }^{23}$ is a clinically relevant phenomenon that precedes drug-seeking behavior and has a potential to trigger relapse. ${ }^{24,25}$ Craving has been shown to predict lapse to opioid use in patients receiving treatment for $\mathrm{OUD},{ }^{26,27}$ and a recent study reported that among 
patients treated for OUD, those with chronic pain had higher opioid craving. ${ }^{28}$ Very relevant to the management of OUD is the abnormal pain sensitivity, associated with craving, persisting for months following abstinence, ${ }^{29}$ which may adversely impact the treatment outcomes and contribute to relapse.

Opioids remain the most powerful and effective medications for the treatment of chronic pain associated with various medical illnesses. ${ }^{30}$ First reported in patients receiving repeated morphine injections, ${ }^{31}$ a paradoxical state of heightened pain sensitivity called opioid-induced hyperalgesia $(\mathrm{OIH})$ emerges following chronic opioid therapy, potentially as a consequence of neuroadaptations in the nociceptive pathways. $^{32,33}$ In this subset of the population, OUD is highly prevalent and even higher doses of opioids are ineffective in controlling pain, a phenomenon collectively referred to as opioid overuse pain syndrome. ${ }^{34-36} \mathrm{OIH}$ has also been documented after rapid titration with intravenous morphine in cancer patients. ${ }^{37}$ Studies have shown that one in five cancer patients is at significant risk of developing OUD, and this risk is much higher when combined with alcohol and smoking. ${ }^{38}$ Although $\mathrm{OIH}$ and tolerance appear to share similar symptoms (i.e., reduced analgesic response to opioid treatment), OIH distinctly differs from tolerance. Whereas an increase in opioid doses may overcome tolerance, this similar approach might intensify pain intensity in a patient with OIH. ${ }^{32}$ Furthermore, in patients receiving opioid medication for chronic pain, the self-reported craving has been reported to predict aberrant use of prescription opioids. ${ }^{39}$ In short, chronic opioid therapy may induce a state of increased pain intensity, which may facilitate opioid overuse/misuse and development of OUD.

OUD and chronic pain may be best framed as a syndrome. Furthermore, certain psychiatric pathologies are frequently comorbid with chronic pain, which can further intensify pain symptoms. ${ }^{40,41}$ As a result, in patients receiving opioid agonist therapy for chronic pain and/or OUD, undertreated or untreated pain conditions may have adverse consequences. ${ }^{28}$ Therefore, patients receiving OAAT for OUD should be screened for pain conditions, and chronic pain must be considered as an additional target for treatment. This, however, could be challenging due to the lack of well-accepted and specific protocols to assess and treat chronic pain in OUD patients receiving OAAT. Furthermore, even wellexperienced practitioners express concerns over diagnostic and clinical decision making in the management of chronic pain comorbid with OUD. ${ }^{42-44}$ A significant disparity in pain management under these conditions may also stem from prioritizing treatment for either OUD or chronic pain. ${ }^{42}$ It is important to understand that OUD and chronic pain may be a result of neuroadaptations in the nociceptive circuitry following prolonged opioid exposure. Insufficient understanding of this interplay coupled with inadequate management of either condition could contribute to relapse and treatment failure. Considering this complex, intertwined relationship between chronic pain and OUD, a multidisciplinary approach of treating both the states is warranted. Providing necessary training, which emphasizes the critical interaction among chronic pain, opioid dependence and tolerance, could equip clinicians to address these unmet needs. In the case, providing a comprehensive treatment plan is not feasible in one clinical setting; OUD treatment providers must work closely with pain clinicians to ensure that both chronic pain and addiction components are addressed completely. In addition, managing comorbid psychiatric complications that may regulate chronic pain state may be necessary to maximize treatment benefits. Further research is necessary to evaluate novel screening, protocols and improved medications to treat chronic pain in OUD patients receiving OAAT. Although challenging, an appropriate evaluation and treatment strategy that adequately treats pain conditions in this population may hold promise in the successful management of OUD with better outcomes.

\section{Acknowledgments}

This publication was made possible with partial funding from the NIMHD-RCMI grant number 5G12MD007595 from the National Institute on Minority Health and Health Disparities and the NIGMS-BUILD grant number 8UL1GM118967 to SS. This publication was also made possible by the Louisiana Cancer Research Consortium. University of Toledo provided start-up funds (F-110760) to AKT. We thank Ms. Charisse Montgomery (UT), Dr. Thomas J Maestri (XULA) and Ms. Anna Smith (XULA) for the critical review of this manuscript. The contents are solely the responsibility of the authors and do not necessarily represent the official views of NIMHD.

\section{Disclosure}

The authors report no conflicts of interest in this work.

\section{References}

1. Rosenblum A, Marsch LA, Joseph H, Portenoy RK. Opioids and the treatment of chronic pain: controversies, current status, and future directions. Exp Clin Psychopharmacol. 2008;16(5):405-416.

2. Hall AJ, Logan JE, Toblin RL, et al. Patterns of abuse among unintentional pharmaceutical overdose fatalities. JAMA. 2008;300(22):2613-2620.

3. Manchikanti L, Singh A. Therapeutic opioids: a ten-year perspective on the complexities and complications of the escalating use, abuse, and nonmedical use of opioids. Pain Physician. 2008;11(2 suppl):S63-S88.

4. National Center for Health Statistics CW. Overdose Death Rates: Trends and Statisitics. https://www.drugabuse.gov/related-topics/ trends-statistics/overdose-death-rates. Accessed September 18, 2016. 
5. Rudd RA, Aleshire N, Zibbell JE, Gladden RM. Increases in Drug and opioid overdose deaths - United States, 2000-2014. MMWR Morb Mortal Wkly Rep. 2016;64(50-51):1378-1382.

6. Wilkerson RG, Kim HK, Windsor TA, Mareiniss DP. The opioid epidemic in the United States. Emerg Med Clin North Am. 2016;34(2):e1-e23.

7. Centers for Disease Control and Prevention. Prevention Status Reports. http://www.cdc.gov/psr/overview.html.

8. American Psychiatric Association. DSM 5. American Journal of Psychiatry; 2013. http://doi.org/10.1176/appi.books.9780890425596.744053. Accessed April 14, 2016.

9. Burns RM, Pacula RL, Bauhoff S, et al. Policies related to opioid agonist therapy for opioid use disorders: the evolution of state policies from 2004 to 2013. Subst Abus. 2016;37(1):63-69.

10. Bart G. Maintenance medication for opiate addiction: the foundation of recovery. J Addict Dis. 2012;31(3):207-225.

11. Stotts AL, Dodrill CL, Kosten TR. Opioid dependence treatment: options in pharmacotherapy. Expert Opin Pharmacother. 2009;10(11): 1727-1740.

12. Magura S, Rosenblum A. Leaving methadone treatment: lessons learned, lessons forgotten, lessons ignored. Mt Sinai J Med. 2001;68(1):62-74

13. Davstad I, Stenbacka M, Leifman A, Beck O, Korkmaz S, Romelsjö A. Patterns of illicit drug use and retention in a methadone program: a longitudinal study. J Opioid Manag. 2007;3(1):27-34.

14. Smyth BP, Barry J, Keenan E, Ducray K. Lapse and relapse following inpatient treatment of opiate dependence. Ir Med J. 2010;103(6): 176-179.

15. Rosenblum A, Joseph H, Fong C, et al. Prevalence and characteristics of chronic pain among chemically dependent patients in methadone maintenance and residential treatment facilities. JAMA. 2003;289(18):2370.

16. Barry DT, Beitel M, Garnet B, Joshi D, Rosenblum A, Schottenfeld RS. Relations among psychopathology, substance use, and physical pain experiences in methadone-maintained patients. J Clin Psychiatry. 2009;70(9):1213-1218.

17. Barry DT, Savant JD, Beitel M, et al. Pain and associated substance use among opioid dependent individuals seeking office-based treatment with buprenorphine-naloxone: a needs assessment study. Am J Addict. 2013;22(3):212-217.

18. Chakrabarti A, Woody GE, Griffin ML, Subramaniam G, Weiss RD. Predictors of buprenorphine-naloxone dosing in a 12-week treatment trial for opioid-dependent youth: secondary analyses from a NIDA Clinical Trials Network study. Drug Alcohol Depend. 2010;107(2-3):253-256.

19. Larson MJ, Paasche-Orlow M, Cheng DM, Lloyd-Travaglini C, Saitz R, Samet JH. Persistent pain is associated with substance use after detoxification: a prospective cohort analysis. Addiction. 2007;102(5):752-760.

20. Caldeiro RM, Malte CA, Calsyn DA, et al. The association of persistent pain with out-patient addiction treatment outcomes and service utilization. Addiction. 2008;103(12):1996-2005.

21. Dhingra L, Perlman DC, Masson C, et al. Longitudinal Analysis of Pain and Illicit Drug Use Behaviors in Outpatients on Methadone Maintenance. 2015;149:285-289. doi:10.1016/j.drugalcdep.2015.02.007.

22. Fox AD, Sohler NL, Starrels JL, Ning Y, Giovanniello A, Cunningham CO. Pain is not associated with worse office-based buprenorphine treatment outcomes. Subst Abus. 2012;33(4):361-365.

23. Rosenberg H. Clinical and laboratory assessment of the subjective experience of drug craving. Clin Psychol Rev. 2009;29(6):519-534.

24. Childress AR, Mozley PD, McElgin W, Fitzgerald J, Reivich M, O’Brien CP. Limbic activation during cue-induced cocaine craving. Am J Psychiatry. 1999;156(1):11-18.

25. Grimm JW, Hope BT, Wise RA, Shaham Y. Neuroadaptation. Incubation of cocaine craving after withdrawal. Nature. 2001;412(6843):141-142.
26. Tsui JI, Anderson BJ, Strong DR, Stein MD. Craving predicts opioid use in opioid-dependent patients initiating buprenorphine treatment: a longitudinal study. Am J Drug Alcohol Abuse. 2014;40(2):163-169.

27. McHugh RK, Fitzmaurice GM, Carroll KM, et al. Assessing craving and its relationship to subsequent prescription opioid use among treatmentseeking prescription opioid dependent patients. Drug Alcohol Depend. 2014;145:121-126.

28. Tsui JI, Lira MC, Cheng DM, et al. Chronic pain, craving, and illicit opioid use among patients receiving opioid agonist therapy. Drug Alcohol Depend. 2016;166:26-31.

29. Ren ZY, Shi J, Epstein DH, Wang J, Lu L. Abnormal pain response in painsensitive opiate addicts after prolonged abstinence predicts increased drug craving. Psychopharmacology (Berl). 2009;204(3):423-429.

30. Trang T, Al-Hasani R, Salvemini D, Salter MW, Gutstein H, Cahill CM. Pain and poppies: the good, the bad, and the ugly of opioid analgesics. J Neurosci. 2015;35(41):13879-13888.

31. Kayan S, Woods LA, Mitchell CL. Morphine-induced hyperalgesia in rats tested on the hot plate. J Pharmacol Exp Ther. 1971;177(3):509-513.

32. Lee M, Silverman SM, Hansen H, Patel VB, Manchikanti L.A comprehensive review of opioid-induced hyperalgesia. Pain Physician. 2011;14(2): 145-161.

33. Roeckel LA, Le Coz GM, Gavériaux-Ruff C, Simonin F. Opioid-induced hyperalgesia: cellular and molecular mechanisms. Neuroscience. Epub 2016 Jun 23

34. Sullivan MD, Edlund MJ, Fan MY, Devries A, Brennan Braden J, Martin BC. Risks for possible and probable opioid misuse among recipients of chronic opioid therapy in commercial and medicaid insurance plans: the TROUP Study. Pain. 2010;150(2):332-339.

35. Boscarino JA, Rukstalis MR, Hoffman SN, et al. Prevalence of prescription opioid-use disorder among chronic pain patients: comparison of the DSM-5 vs. DSM-4 diagnostic criteria. J Addict Dis. 2011;30(3):185-194.

36. Mehendale AW, Goldman MP, Mehendale RP. Opioid overuse pain syndrome (OOPS): the story of opioids, prometheus unbound. J Opioid Manag. 9(6):421-438.

37. Mercadante S, Ferrera P, Arcuri E, Casuccio A. Opioid-induced hyperalgesia after rapid titration with intravenous morphine: Switching and retitration to intravenous methadone. Ann Palliat Med. 2012;1(1):10-13.

38. Rauenzahn S, Del Fabbro E. Opioid management of pain: the impact of the prescription opioid abuse epidemic. Curr Opin Support Palliat Care. 2014;8(3):273-278.

39. Wasan AD, Butler SF, Budman SH, et al. Does report of craving opioid medication predict aberrant drug behavior among chronic pain patients? Clin J Pain. 2009;25(3):193-198.

40. Ramírez-Maestre C, López Martínez AE, Zarazaga RE. Personality characteristics as differential variables of the pain experience. $J$ Behav Med. 2004;27(2):147-165.

41. Carcoba LM, Contreras AE, Cepeda-Benito A, Meagher MW. Negative affect heightens opiate withdrawal-induced hyperalgesia in heroin dependent individuals. J Addict Dis. 2011;30(3):258-270.

42. Berg KM, Arnsten JH, Sacajiu G, Karasz A. Providers' experiences treating chronic pain among opioid-dependent drug users. J Gen Intern Med. 2009;24(4):482-488.

43. Longo DL, Volkow ND, McLellan AT. Opioid Abuse in chronic pain misconceptions and mitigation strategies. N Engl J Med. 2016;374(13): 1253-1263.

44. Keller CE, Ashrafioun L, Neumann AM, Van Klein J, Fox CH, Blondell RD. Practices, perceptions, and concerns of primary care physicians about opioid dependence associated with the treatment of chronic pain. Subst Abus. 2012;33(2):103-113. 
Dove Medical Press encourages responsible, free and frank academic debate. The content of the Journal of Pain Research 'Editorial' section does not necessarily represent the views of Dove Medical Press, its officers, agents, employees, related entities or the Journal of Pain Research editors. While all reasonable steps have been taken to confirm the content of each Editorial, Dove Medical Press accepts no liability in respect of the content of any Editorial, nor is it responsible for the content and accuracy of any Editorial.

Journal of Pain Research

Publish your work in this journal

The Journal of Pain Research is an international, peer reviewed, open access, online journal that welcomes laboratory and clinical findings in the fields of pain research and the prevention and management of pain. Original research, reviews, symposium reports, hypothesis formation and commentaries are all considered for publication. 